\title{
Tubularized Incised Plate (TIP) Repair Improves Outcome of Hypospadias Repair
}

\begin{abstract}
Keywords: Hypospadias; Boys; Reconstruction; Outcome; Complications

Abstract

Aim of the study: To analyze if a change of surgical method affects the complication rate in hypospadias surgery.

Methods: Over a period of six years, the Mathieu procedure was replaced by the Tubularized Incised Plate (TIP) repair as the most favored method of grade 1-2 hypospadias repair at our clinic. All operated boys were prospectively registered during those years. Two three-year-periods were outlined with equal number of patients: 2010 2012 and 2013-2015, but with the preferred method of repair reversed. The end point was any complication calling for a reoperation.

Main results: In the first period 69 boys were operated whereo 50 Mathieu and 19 TIP. 35 (51\%) boys required a reoperation. In the second period 73 boys were operated whereof 19 Mathieu and 54 TIP. $15(21 \%)$ required a reoperation, $P<0.01$.

Research limitations: The study is prospective but the technique preferred was the choice of the surgeon. Follow up will be continued due to acknowledged accumulation of complications over time.

Conclusion: Method seems to matter. Advantage TIP. We are not aware of any other prospective study following a shift in paradigm and at the same time comparing these two procedures for hypospadias repair.
\end{abstract}

\section{Introduction}

\section{Background}

Until 2012 the predominant method of hypospadias repair at our clinic was the Mathieu procedure [1]. We then successively replaced this method with the Tubularized Incised Plate (TIP) repair [2-4], which has become the most used technique worldwide. The reason for the change was the high complication rates in our series $[5,6]$ as well as in others reported in the literature [7].

\section{Purpose}

To evaluate if a shift in surgical method decreases the complication rate in hypospadias surgery

\section{Material and Methods}

\section{Design/methodology/approach}

Every boy consecutively undergoing grade 1 or 2 hypospadias reconstruction at our clinic was registered prospectively from early 2010 until the end of 2015.

This period was split into two equal halves mirroring the shift in paradigm. The choice of method was up to the surgeon. The reasons for changing the method were a high frequency of postoperative complications and recommendation from a colleague from a nearby center. The first period covers the years of 2010-2012 and the second the following three years

\section{Journal of} Surgery

\section{Hans Winberg*, Magnus Anderberg and Einar Arnbjörnsson}

Department of Pediatric Surgery, Skåne University Hospital, Lund, Sweden

*Address for Correspondence

Hans Winberg, Department of Pediatric Surgery, Skåne University Hospital, Lund, Sweden, E-mail: winberghans@gmail.com

Submission: 24 October, 2016

Accepted: 28 November, 2016

Published: 03 December, 2016

Copyright: $\odot 2016$ Winberg $\mathrm{H}$, et al. This is an open access article distributed under the Creative Commons Attribution License, which permits unrestricted use, distribution, and reproduction in any medium, provided the original work is properly cited.

2013-2015. There were no changes in the suture material used. The operative interventions were performed by two pediatric surgeons with 24 and 11 years' experience in hypospadias surgery respectively.

All complications were handled by the same team of surgeons.

The main outcome measure was any complication calling for a reoperation: a fistula, rupture and/or stricture, during the period from the operative intervention until August 2016, the endpoint of the study.

\section{The Methods of Operation}

\section{Mathieu repair}

During a Mathieu procedure [1] a skin flap based towards the meatus [8] is turned 180 degrees and sutured into incision on both sides of the glanular groove and along the tip. Curvature is corrected when present. The prepuce is then partially divided and mobilized to create an outer layer.

\section{Tubularized Incised Plate repair (TIP)}

When a TIP repair [2] is performed a U-shaped skin incision is made along the edges of the urethral plate and the penis is degloved. The urethral plate is widened by a midline incision along its length and then tubularized over a stent. A pedicle of subcutaneous tissue is dissected from the ventral or dorsal penile skin and used to cover the neourethra. Finally, the glanular wings, mucosal collar and ventral penile skin are closed in the midline.

\section{Statistical considerations}

A clinical significant relevance was set at a decrease in the complication rate with $20 \%$ units. The intention was to study independent cases and controls with 1 control(s) per case. Prior data indicate that the probability of exposure among controls is 0.3 . If the true probability of exposure among cases is 0.1 , we will need to study 72 case patients and 72 control patients to be able to reject the null hypothesis that the exposure rates for case and controls are equal with probability (power) 0.8 . The Type I error probability associated with this test of this null hypothesis is 0.05 . We will use a continuitycorrected chi-squared statistic or Fisher's exact test to evaluate this null hypothesis. 
Citation: Winberg H, Anderberg M. Arnbjörnsson E. Tubularized Incised Plate (TIP) Repair Improves Outcome of Hypospadias Repair. J Surgery. 2016;4(2): 4.

\section{Ethical considerations}

This study was performed according to the Helsinki declaration and approved by the Regional Ethical Review Board (registration number 2010/49). The study protocol was designed to meet the legislative documentation required by the country of origin.

The data were anonymized prior to calculations, and are presented in such a manner that it is impossible to identify or link to any specific individual. Therefore, it was not necessary to obtain approval from the individual patient's guardians to conduct this study. It will not be possible to go back and trace or identify any of the participants.

Since this data was retrospectively collected from a prospectively collected database, the treatment plan of each patient was not altered. All evaluations, treatments, and procedures described in this report met the standard of care and were conducted at a tertiary center for pediatric surgery. No protocols were exercised that would have required appropriate informed consent or approval of an institutional review board. The risk of harming the patients in this study in a physical, social or psychological manner was nonexistent.

\section{Results}

The findings are summarized in Table 1. During the study 142 boys were operated on, 69 and 73 boys in the first and second period respectively. In the first period 50 (72\%) of the boys underwent the Mathieu procedure compared to the second period were only 19 (26\%) were selected for this method. The corresponding numbers for the TIP procedure were $19(28 \%)$ and $54(74 \%)$. In the first period
$35(51 \%)$ of the boys needed another operation compared to only 15 $(21 \%)$ in the second, $\mathrm{P}<0.01$, Table 1.

The duration of follow up was longer in the first period, Table 1. The duration of the follow-up was influenced by emigration to other countries and in some cases caretaking at another hospital in the region or by their own parents with urological competence.

The most common postoperative complication was an urethra cutaneous fistula, Table 1. The time of the reoperations was median 15 months (range 2-72) postoperatively and was not significantly different between the groups from the two periods.

\section{Discussion}

The results of the study showed that the change in the method used for the hypospadias reconstruction in our hands significantly improved the outcome for the boys undergoing a hypospadias repair. There were no other changes e.g. in surgical method or suture material used during the period studied. The operative interventions were performed by the same team of two experienced pediatric surgeons.

To our knowledge, there are no randomized clinical trials (RTC) comparing different methods for the hypospadias reconstruction in the literature. There are however, studies in the literature comparing the two methods, but with contradicting results [9]. Thus, the conduction of a RTC would be difficult to gain acceptance in an ethical committee since the compared methods should be equal regarding outcome in order to permit a randomization between two groups of children.

Table 1: The rate of complications in 142 boys undergoing hypospadias repair with two different methods, Mathieu vs TIP.

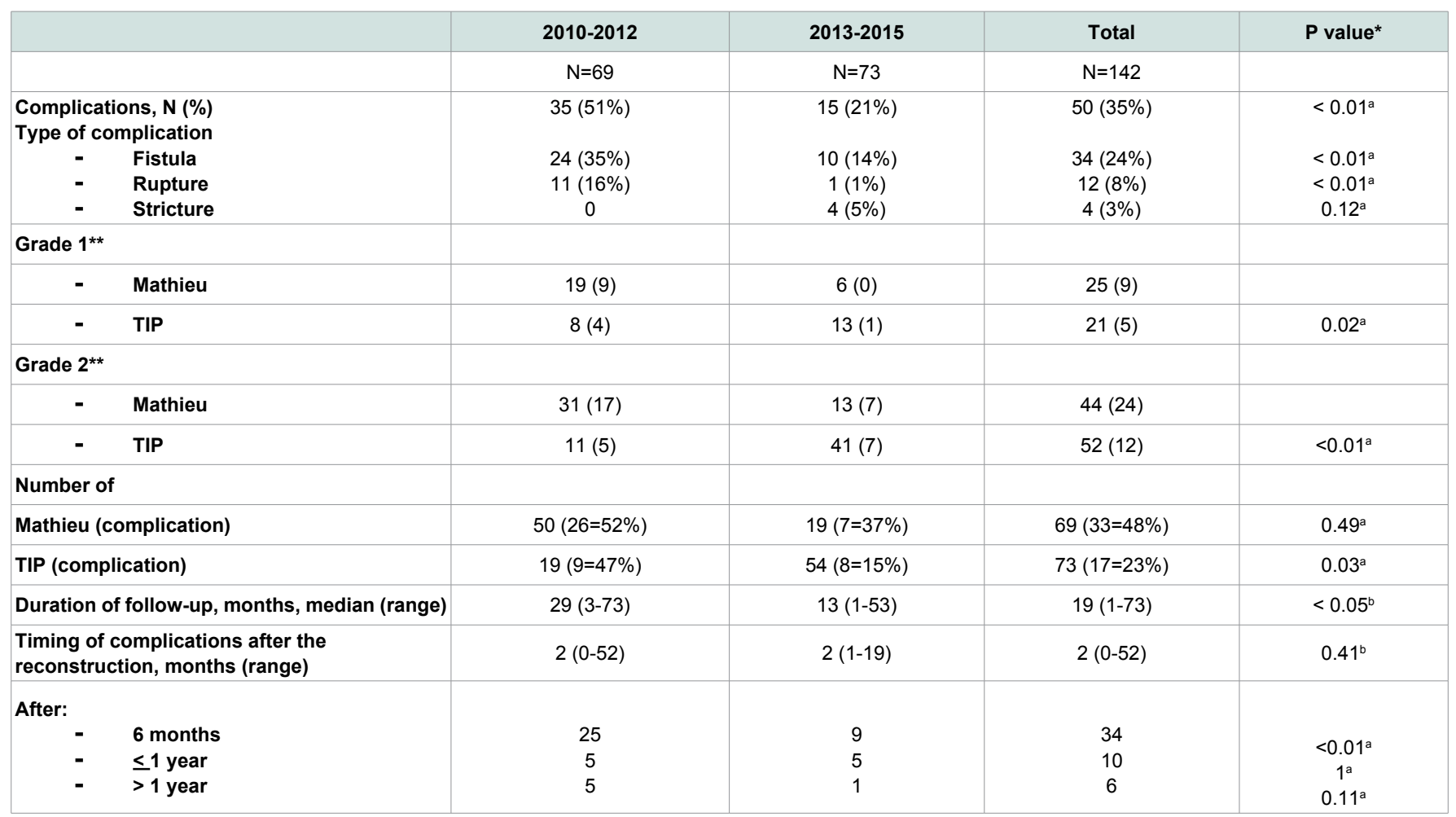

*Statistical method: Fisher's exact test ${ }^{\mathrm{a}}$ and Mann-Whitney $U$ test $^{\mathrm{b}}$.

${ }^{\star *}$ The numbers in brackets are complications, a fistula, a rupture or a stricture 
Citation: Winberg H, Anderberg M. Arnbjörnsson E. Tubularized Incised Plate (TIP) Repair Improves Outcome of Hypospadias Repair. J Surgery. 2016;4(2): 4.

ISSN: $2332-4139$

The originality and value of our study is that it is a prospective study following a shift in paradigm and comparing two procedures for hypospadias repair.

Thirty-five percent of all the 142 boys in our series had a complication. This is in accordance with the recent literature with complication rates ranging from 7 to $50 \%[5,6,10-18]$. Thus, it is obvious that an effort has to be made with the intention to improve the outcome of hypospadias surgery. This study is an example of an effort to improve the outcome.

The reason for the change of method of operation to the TIP was the poor results with the Mathieu method before the study started. During the first phase there was no difference between the outcomes of the two methods used for reconstruction. Insignificant better results after the TIP reconstruction motivated further and increased used of TIP. The better results with TIP in phase 2 can be attributed to more experience gained by the surgeons. As shown in Table 1 the complications of the Mathieu repair are reduced from phase 1 to 2 ( $52 \%$ vs $37 \%)$. This difference is not significant $(p=0.49$ ).

The TIP method appears to be more embryological and utilizing the natural conditions for the correction of the deformity [19]. The technique of TIP repair was not modified during the time period studied. There were no selection criteria for the TIP repair other than surgeon's preference. The urethral plate was not used as parameter to select the type of repair. Another advantage is to have fewer basic methods to adapt, instead of choosing among many when facing each patient, since the TIP procedure can be used for more variants of hypospadias than the Mathieu procedure. A learning curve may be tracked in the series in our study.

The duration of follow-up in our series is comparable to what is reported in the literature. A randomly selected recent retrospective case series [19] reported that the median follow-up was 27 months, range between 6 weeks and 9 years. The majority of papers did not address the issue of if the boys were lost to follow-up or excluded from the study. It has to be assumed that the follow-up rate usually is $100 \%$ as is the case in our group of boys [20].

In a recent report 17 articles of hypospadias repair using the TIP method were reviewed. All studies performed surgery and repair on the basis of the Snodgrass's method; however, some introduced modifications to the method. The prevalence of complications in repeated TIP surgery was 11 to $33 \%$ and the most prevalent complication in different studies was fistulae. Based on the metaanalysis, the overall estimation of complications was $22 \%$ (95\% CI: 18 to 26) [21]. These results are comparable to our complication rate after the shift in the method of operation.

\section{The Research Limitations, Weakness and Strengths}

This study is limited by not being randomized. Thus, a bias was introduced in the case selection for the type of repair. The surgeon's preference decided the selection of the type of repair. We cannot exclude that boys with poor urethral plates were considered unsuitable for TIP repair and that the surgeon chose to perform a Mathieu repair in those cases.

The follow up time is naturally shorter in the second group and we know from other studies [10] that complications can assemble over time.

The strengths of this study are that it is prospective and that all the boys had undergone the same standardized hypospadias reconstructive operation by the same team of surgeons. Through our follow up program it will be possible to make a fairer comparison in the years to come. The inhabitants in Sweden are provided with free health care when needed. Therefore, any drop out due to socioeconomic effects is unlikely.

\section{Conclusion}

The results show that the method matters, at least in our hands. This applies also to others. We find it difficult to ignore the starching results in our study, which supports our measures taken for improving the outcome of distal hypospadias repair.

\section{Practical Implications}

The findings of this study are of value for use in parents' information in preoperative consultations before hypospadias surgery. Furthermore, comparison with other centers is made possible.

\section{References}

1. Mathieu $P$ (1932) Treatment in one time of hypospadias balanique and juxta balanique. J Chir (Paris) 39: 481-484.

2. Snodgrass WT (1999) Tubularized incised plate hypospadias repair: indications, technique, and complications. Urology 54: 6-11.

3. Snodgrass W, Bush N (2016) TIP hypospadias repair: A pediatric urology indicator operation. J Pediatr Urol 12: 11-18.

4. Snodgrass W, Bush N (2015) Surgery for primary proximal hypospadias with ventral curvature $>30^{\circ}$. Curr Urol Rep 16: 69 .

5. Winberg $H$, Westbacke $G$, Ekmark AN, Anderberg M, Arnbjörnsson $E$ (2014) The complication rate after hypospadias repair and correlated preoperative symptoms. Open J Urol 4: 155-162.

6. Winberg $H$, Jinhage $M$, Träff H, Salö M, Westbacke $G$, et al. (2016) Urinary tract infection in boys with hypospadias. MOJ Surg 3: 00045.

7. Cimador M, Vallasciani S, Manzoni G, Rigamonti W, De Grazia E, et al (2013) Failed hypospadias in paediatric patients. Nat Rev Urol 10: 657-666.

8. Boddy SA, Samuel M (2000) Mathieu and 'V' incision sutured (MAVIS) results in a natural glanular meatus. J Pediatr Surg 35: 494-496.

9. Wilkinson DJ, Farrelly P, Kenny SE (2012) Outcomes in distal hypospadias: a systematic review of the Mathieu and tubularized incised plate repairs. $J$ Pediatr Urol 8: 307-312.

10. Ekmark AN, Svensson H, Arnbjörnsson E, Hansson E (2013) Postpubertal examination after hypospadias repair is necessary to evaluate the success of the primary reconstruction. Eur J Pediatr Surg 23: 304-311.

11. Snodgrass WT, Koyle MA, Baskin LS, Caldamone AA (2006) Foreskin preservation in penile surgery. J Urol 176: 711-714.

12. Aslam R, Campbell K, Wharton S, Bracka A (2013) Medium to long term results following single stage Snodgrass hypospadias repair. J Plast Reconstr Aesthet Surg 66: 1591-1595.

13. Spinoit AF, Poelaert F, Groen LA, Van Laecke E, Hoebeke P (2013) Hypospadias repair at a tertiary care center: long-term followup is mandatory to determine the real complication rate. J Urol 189: 2276-2281.

14. Prat D, Natasha A, Polak A, Koulikov D, Prat O, et al. (2012) Surgical outcome of different types of primary hypospadias repair during three decades in a single center. Urology 79: 1350-1353.

15. Elganainy EO, Abdelsalam YM, Gadelmoula MM, Shalaby MM (2010) 
Citation: Winberg H, Anderberg M. Arnbjörnsson E. Tubularized Incised Plate (TIP) Repair Improves Outcome of Hypospadias Repair. J Surgery. 2016;4(2): 4.

ISSN: $2332-4139$

Combined mathieu and snodgrass urethroplasty for hypospadias repair: a prospective randomized study. Int J Urol 17: 661-665.

16. Sarhan O, Saad M, Helmy T, Hafez A (2009) Effect of suturing technique and urethral plate characteristics on complication rate following hypospadias repair: a prospective randomized study. J Urol 182: 682-685.

17. Pfistermuller KL, McArdle AJ, Cuckow PM (2015) Meta-analysis of complication rates of the tubularized incised plate (TIP) repair. J Pediatr Urol 11: 54-59.

18. Hardwicke JT, Bechar JA, Hodson J, Osmani O, Park AJ (2015) Fistula after single-stage primary hypospadias repair - a systematic review of the literature. J Plast Reconstr Aesthet Surg 68: 1647-1655.

19. Snodgrass W, Bush N (2014) Recent advances in understanding/ management of hypospadias. F1000Prime Rep 6: 101.

20. Springer A (2014) Assessment of outcome in hypospadias surgery - a review. Front Pediatr 2: 2.

21. Mousavi SA, Aarabi M (2014) Tubularized incised plate urethroplasty for hypospadias reoperation: a review and meta-analysis. Int Braz J Urol 40: 588-595.

\section{Acknowledgements}

We are grateful to HåkanLövkvist, biostatistician at the Competence Centre for Clinical Research, Skåne University Hospital, Lund, Sweden, for statistical advice.

This manuscript has been edited by native English-speaking medical experts from BioMed Proofreading LIC. 